\title{
Partial Thyroidectomy
}

National Cancer Institute

\section{Source}

National Cancer Institute. Partial Thyroidectomy. NCI Thesaurus. Code C51921.

Surgery involving partial excision of the thyroid gland. 